\title{
AOR
}

Selected Papers of \#AolR2020:

The 22nd Annual Conference of the Association of Internet Researchers Virtual Event / 13-16 Oct 2021

\section{MASK NARRATIVES PROMOTED BY ANTI-VACCINATION ACCOUNTS ON INSTAGRAM PRIOR TO THE COVID-19 PANDEMIC}

Kolina Koltai

The University of Washington

Iva Grohmann

The University of Washington

Devin T. Johnson

The University of Washington

Samantha Rondini

The University of Washington

Ella R. Foley

The University of Washington

\section{Introduction \& Context}

There has been widespread debate over the efficacy and use of masks and face coverings during the COVID-19 pandemic stemming from the proliferation of misinformation online. While there are many different actors that have contributed to the conflicting public health messages, the anti-vaccination movement has been particularly central in spreading health misinformation (Burki, 2020).

There have been numerous studies conducted exploring anti-vaccination narratives (Kata, 2012) and the information behaviors (Koltai, 2020) of the anti-vaccination movement online. We know that the spread of vaccine misinformation online is a leading contributor to vaccine hesitancy. The online groups and accounts which focus their efforts on spreading vaccine misinformation through varied rhetorical techniques (e.g., pseudo-scientific claims) (Moran et al., 2016) have also been responsible for spreading other health misinformation.

In this work, we investigate mask-wearing narratives of the anti-vaccination community prior to COVID-19's onset among Instagram accounts that have repeatedly shared antivaccination content. During the course of the global pandemic, there has been 
demonstrated overlap between the anti-mask and anti-vaccine communities (Taylor \& Asmundson, 2021). Given the anti-vaccination community's promotion of false and misleading content about masks during the pandemic, this prompts the question of whether they always held this negative sentiment about masks. Given that masks can be an alternative to vaccines to prevent disease transmission, we believe it is possible that mask-wearing could've been a supported behavior in the movement prior to COVID-19. Therefore, the overarching research question that guides this preliminary work is: What were the mask narratives promoted by anti-vaccination accounts prior to the COVID-19 pandemic?

\section{Methods}

We were interested in identifying Instagram users who had previously shared content about masks and vaccines prior to the pandemic. Using CrowdTangle, a public insight tool from Facebook, we collected public Instagram posts that were shared between October 1, 2017, to December 31, 2019. These dates were chosen because we wanted to cover posts that would have happened over a normal flu season in the US (starting in October) and avoid any overlap with the pandemic. Any post from a public account that included the word "mask" and either "flu," "flue," "vaccine," or "nurse" was collected. We had exclusion terms like "Halloween" to remove noise. This resulted in 1,010 posts from 793 accounts. Foreign language posts were not included in the analysis. These posts were collected on December 29, 2020.

The research team hand-coded the posts to determine if they were in-scope (e.g., about medical masks). From this set, the team then evaluated the remaining accounts for their vaccine stance. We only included Instagram users who expressed negative sentiments about vaccines in our dataset. We were left with 30 anti-vaccine accounts and 44 posts mentioning face masks prior to 2020. Using thematic analysis (Braun \& Clarke, 2006), we identified several key themes.

\section{Findings}

\section{Mask Efficacy}

The theme of mask efficacy centers around the intended uses and benefits of wearing masks. Posts often advocated for the efficacy of masks as an argument that they were more effective than vaccines at preventing the spread of seasonal flu and stressed the low efficacy rate of the seasonal flu shot in previous years. Further, some posts around mask efficacy highlight that wearing a mask can signal that vaccines do not work. These posts describe beliefs that masks can protect yourself and others from getting sick.

\footnotetext{
Masks as a form of control

While masks were discussed positively when it came to efficacy, there was some negative sentiment around masks, primarily stemming from mandates. In many healthcare settings, an employee must either agree to a seasonal flu vaccination or wear a mask for the duration of the flu season. While masks were seen as an alternative to vaccination, some accounts shared content about how they felt they were forced to wear a mask or felt discriminated against due to their opposing vaccine stance. There was also discussion about masks being used as a way to force vaccines, as masks were seen as socially undesirable and difficult to wear for long periods.
} 
Societal Perceptions

Mask posts would also cover the social perceptions of mask-wearing. Some users believed mask-wearing disclosed an individual's anti-vaccination stance and perceived those who wore masks as more intelligent or brave. Masks served as "a conversation piece" for healthcare workers to spread vaccine misinformation. However, some posts acknowledged the social drawbacks of mask-wearing, namely that others were more likely to assume they were sick or infectious and may be more likely to avoid them.

\section{Physical experience wearing masks}

Although slightly more uncommon, several posts mentioned the negative physical experiences associated with wearing a mask. These posts expressed the discomfort of breathing in masks and for wearing them for extended periods. Other posts were more specific in their criticism, such as that it made reading lips more difficult for Deaf or Hard of Hearing individuals. However, these posts generally framed the difficulty of maskwearing as worthwhile to avoid vaccination.

\section{Conclusions, Limitations, and Future work}

Overall, posts about masks by vaccine opposed Instagram accounts were generally positive prior to the COVID-19 pandemic. Most interestingly, masks were often praised for their efficacy which is counter to narratives about masks shared by anti-vaccine accounts during the pandemic. Further, the themes we saw about masks were consistent with anti-vaccine themes. Specifically, centering the conversation about masks around freedom is consistent with anti-vaccination narratives (Koltai, 2020). Notably, during the pandemic, there was a deluge of pseudo-science about the ineffectiveness of masks pushed online. Knowing that there was a drastic shift in mask sentiment and narratives about mask-wearing in this community suggests that it is often less about the science of masks and more about the narrative of freedom and choice. Arguably, this can inform future interventions to increase positive sentiment about masks and vaccines in those who are vaccine hesitant by emphasizing that it gives them freedom instead of limiting it.

The limitations of this work are that we collected posts at the end of 2020. Instagram, like other social media platforms, has been removing posts and accounts that violated their content policies. We, unfortunately, would not have any of these accounts in our data set. Future planned work involves comparing mask sentiments before and during the pandemic by anti-vaccine accounts on multiple social media platforms and evaluating the accounts and sources that pushed for this shift in mask sentiment.

\section{References}

Burki, T. (2020). The online anti-vaccine movement in the age of COVID-19. The Lancet Digital Health, 2(10), e504-e505.

Braun, V. \& Clarke, V. (2006). Using thematic analysis in psychology. Qualitative research in psychology, 3(2), 77-101. 
Kata, A. (2012). Anti-vaccine activists, Web 2.0, and the postmodern paradigm-An overview of tactics and tropes used online by the anti-vaccination movement. Vaccine, 30(25), 3778-3789.

Koltai, K. (2020). Human values and Scientific Controversies: Studying vaccine information behavior on social networking sites. [Doctoral dissertation, University of Texas at Austin].

Moran, M. B., Lucas, M., Everhart, K., Morgan, A., \& Prickett, E. (2016). What makes anti-vaccine websites persuasive? A content analysis of techniques used by antivaccine websites to engender anti-vaccine sentiment. Journal of Communication in Healthcare, 9(3), 151-163.

Taylor, S. \& Asmundson, G. J. G. (2021). Negative attitudes about facemasks during the COVID-19 pandemic: The dual importance of perceived ineffectiveness and psychological reactance. PLOS ONE, 16(2). e0246317 